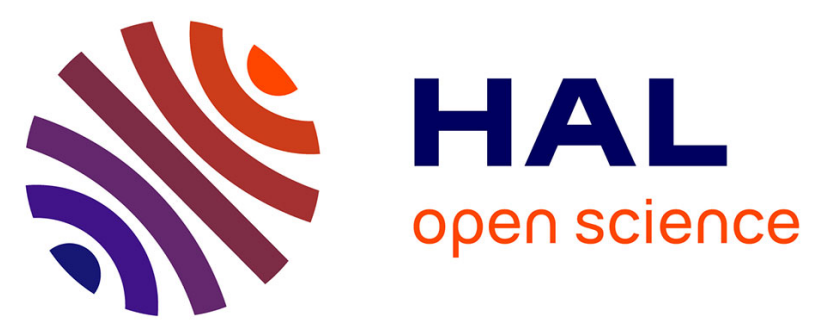

\title{
A fast auto-adaptive method to study the frequency behavior of radiating structures, applied to automatic determination of resonances
}

Pierre Dubois, Jean-Pierre Damiano, Claude Dedeban, Jean-Paul Zolesio

\section{To cite this version:}

Pierre Dubois, Jean-Pierre Damiano, Claude Dedeban, Jean-Paul Zolesio. A fast auto-adaptive method to study the frequency behavior of radiating structures, applied to automatic determination of resonances. International Symposium on Antennas Technology and Applied Electromagnetics (ANTEM 05), Jun 2005, Saint-Malo, France. pp.386-387, 10.1109/ANTEM.2005.7852067 . hal00125403

\section{HAL Id: hal-00125403 \\ https://hal.science/hal-00125403}

Submitted on 3 Feb 2020

HAL is a multi-disciplinary open access archive for the deposit and dissemination of scientific research documents, whether they are published or not. The documents may come from teaching and research institutions in France or abroad, or from public or private research centers.
L'archive ouverte pluridisciplinaire HAL, est destinée au dépôt et à la diffusion de documents scientifiques de niveau recherche, publiés ou non, émanant des établissements d'enseignement et de recherche français ou étrangers, des laboratoires publics ou privés. 


\title{
A FAST AUTO-ADAPTIVE METHOD TO STUDY THE FREQUENCY BEHAVIOR OF RADIATING STRUCTURES, APPLIED TO AUTOMATIC DETERMINATION OF RESONANCES.
}

\author{
P. Dubois (1), J.-P. Damiano (2), C. Dedeban (1), J.-P. Zolésio (3) \\ (1) France Telecom R\&D, Fort de la Tête de Chien 06320 la Turbie ${ }_{\text {; }}$ France, \\ claude.dedeban@francetelecom.com, pierre.dubois@francetelecom.com \\ (2) Laboratoire d'Electronique, Antennes et Télécommunications, Université de Nice-Sophia Antipolis-CNRS, \\ 250 rue Albert Einstein, 06560 Valbonne, France, damiano@unice.fr \\ (3) CNRS-INRIA, Projet OPALE, 2001 Route des Lucioles, 06560 Valbonne, France, Jean-Paul.Zolesio@inria.fr
}

\section{Abstract}

Solving electromagnetic scattering and radiation problems with moment methods or finite element methods over a large frequency band gives accurate results, but it is often too time-consuming. So we propose a fast auto-adaptive method to optimize the computational electromagnetics solver applied in the case of various radiating and dielectric structures. This method is able to generate the electromagnetic behavior of the antenna with a very few simulations over a broad frequency band.

\section{Introduction}

The cost of computational electromagnetic simulation by the moment method or the finite-element method is expensive because the computer model has to be run for every frequency sample. The user often reduces the number of frequency samples in order to have a moderate computing time. However, with a few number of sample points, physical effects are wrong considered, so the obtained results are not right. All these facts are common to various applications as signal processing, microwave imaging reconstruction and optimization in electromagnetic topics, and so on. In recent years, various interpolation algorithms have been developed and published [1-10], particularly for 2D structures.

For example, in the case of a 3D antenna structure, our aim is to obtain the current flows at the antenna surface over the frequency band using only a limited number of frequency samples, adding specific information based on the knowledge of the derivatives of these current flows [11-12]. Thus we have developed a fast auto-adaptive algorithm to save the computation time and give excellent results.

\section{Modeling}

\subsection{Analysis}

With the frequency derivability results [12] associated to the Huyghens principle for $\mathrm{C}^{2}$ surfaces, and the two derivatives of the Rumsey reaction obtained by a computer algebra system (Maple), we determined the unknown current flows and their derivatives at the antenna surface for a very small set of frequency samples. The expressions of the derivatives of the current flow become more complex when the order of derivation increases and the kernel singularity is never stronger than the original one. Nevertheless the integration of the successive kernels needs specific developments.

Solving numerically the Rumsey reaction, we use a finite element computer code (SR3D of France Télécom R\&D [13]). It is based on an integral equation formulation with a triangular finite element discretization. Thus software SR3D solves an bilinear form equation where a is a bilinear form, $x$ the vector flow, $x^{1}$ the functions tests. By deriving this expression at the order $k$ compared to the pulsation $\omega$ one finds:

$a_{\omega}\left(\left(\frac{\partial x}{\partial \omega}\right)^{k}, x^{t}\right)=\left(\frac{\partial x}{\partial \omega}\right)^{k}\left(x^{t}\right)-\sum_{m=0}^{k-1} k^{m}\left(\frac{\partial}{\partial \omega}\right)^{k-m} a\left(\left(\frac{\partial x}{\partial \omega}\right)^{m}, x^{t}\right)$

For more visibility, we note $\mathbf{X}$ the current flow instead of the notation $x$ above. Thus the derivative the current flow $\left(\mathbf{X}^{\prime}, \mathbf{X}^{\prime \prime}\right)$ are solutions of a matrix system:

A. $\mathbf{X}=\mathbf{B}$

Operator $\mathrm{A}$ is the same one, only the second member changes : B' for $X^{\prime}$ and B" for $X^{\prime \prime}$. It consists of the successive derivatives of $A$ and $B$ and of lower order derivatives of the current flow. After calculating the vector solution $\mathbf{X}$, the derivative operators and the new second member are computed. Then we solve the system. The calculation of the derivatives of the current flow at each frequency requires the one of the successive derivatives of bilinear forms $\mathrm{A}$ and $\mathrm{B}$.

Then, our special adaptive interpolation routine is applied to evaluate the current flow and its two first derivatives on the broad frequency band.

The analysis of the behavior of the interpolation function (interpolated current flows) allows to detect critical points where a small number of new sampling frequency points is needed. So the surface currents can be computed faster (ratio from 1 to 10 ) and we are able to deduce the expressions of the antenna characteristics. 


\subsection{Interpolation}

Interpolating and approximating a function by polynomials or rational functions looks hard if we know only a small number of sample points without other information.

Many kinds of interpolation methods exist, for example : the well known least-square method, the Taylor series, the Newton, Lagrange, Chebyshev and trigonometric polynomial interpolations, and so on. The Padé is a well old formal transformation of the first terms of a serie into a rational function as a Chebyshev or Chebyshev-Padé approximation. The Thiele interpolation is used to derive the rational function interpolating the data points with the use of continued fractions from the sample points. A piecewise polynomial function that can have a locally very simple form and smooth. Splines are very useful for modelling arbitrary functions. A rational approximation is sometimes superior to polynomial one because of their ability to model functions with poles. The degree of the two polynomials are quite difficult to be determined. This approximation is sometimes used by ComputerAided-Design software analysing general planar structures (Model Based Parameter Estimation (MBPE) or Adaptive Frequency Sampling modules). It allows to halve or more the number of the computed points.

We develop an original and flexible auto-adaptive algorithm based on a fifth order piecewise polynomial interpolation associated with the knowledge of the true derivatives of the function. This choice allows to minimize much more the number of frequency samples. For example, we consider a arbitrary test function with peaks. We present a comparison between various interpolation results and our model with 7 points only for a $40 \%$ bandwidth (figure 1) without any refinement. Here a fifth degree polynomial is used in the least square method. So we observe that our theoretical results are in excellent agreement with the original variations.

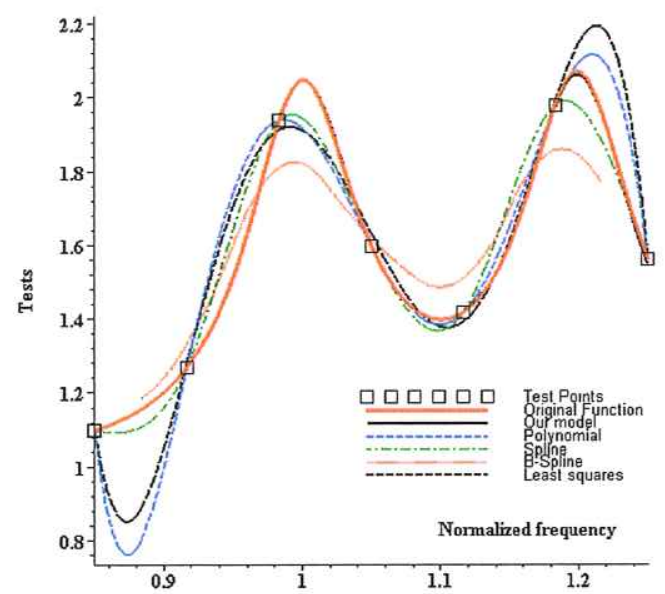

FIG. 1: Comparison between various interpolation results and our model : 7 points only for a $40 \%$ bandwidth
The auto-adaptive algorithm based on the behavior of the $\Psi_{\mathrm{M}}$ function given below :

$\Psi_{M}\left(f_{i}\right)=\frac{1}{M} \sum_{p \in S_{M}(f)}\left|I_{p}(f)\right|^{2}$

$\mathrm{M}$ is the number of considered strongest maxima of the current flow. The value of $M$ can depends on the description of the structure, in terms of freedom degrees number. This algorithm chooses the value of $\mathrm{M}$ and calculates the $\Psi_{\mathrm{M}}$ function.

\section{Results}

\subsection{The antenna structure}

We consider the line-slotted patch antenna structure given in figure 2 . Here, we present some examples of the efficiency of the auto-adaptive algorithm.

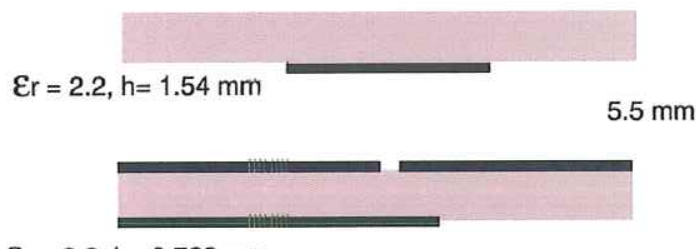

$\varepsilon r=2.2, h=0.782 \mathrm{~mm}$

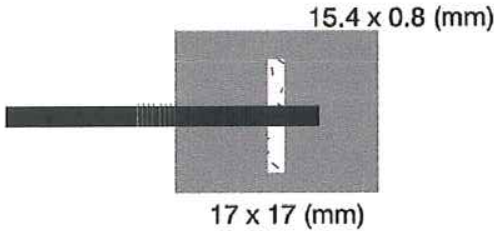

FIG. 2 : The double patch antenna structure (13000 unknowns with the finite element code)

In the figure 3, we can see we start the interpolation process with 7 initial frequency samples only.

The top part of the figure presents the comparison of the average of the twenty strongest values of the modulus of the current flow versus the frequency $(4.5-6.0 \mathrm{GHz})$ for various numerical simulations. The bottom part concerns the quadratic average of the twenty first strongest values of the modulus square of the current flows. The study of these variations are sufficient to give a excellent idea of the behavior of the real current flow.

The black curve is related to the level 0 of the algorithm: i.e. the numerical simulation including interpolation with 7 equidistant frequency samples only (black markers). The dotted line is the case of the first level of refinement ( 2 additional points found by the algorithm). The double dotted line concerns the second level of refinement (3 other new additional points). The $\mathrm{O}$ symbols show the results obtained by the classical SR3D code without any optimization. 

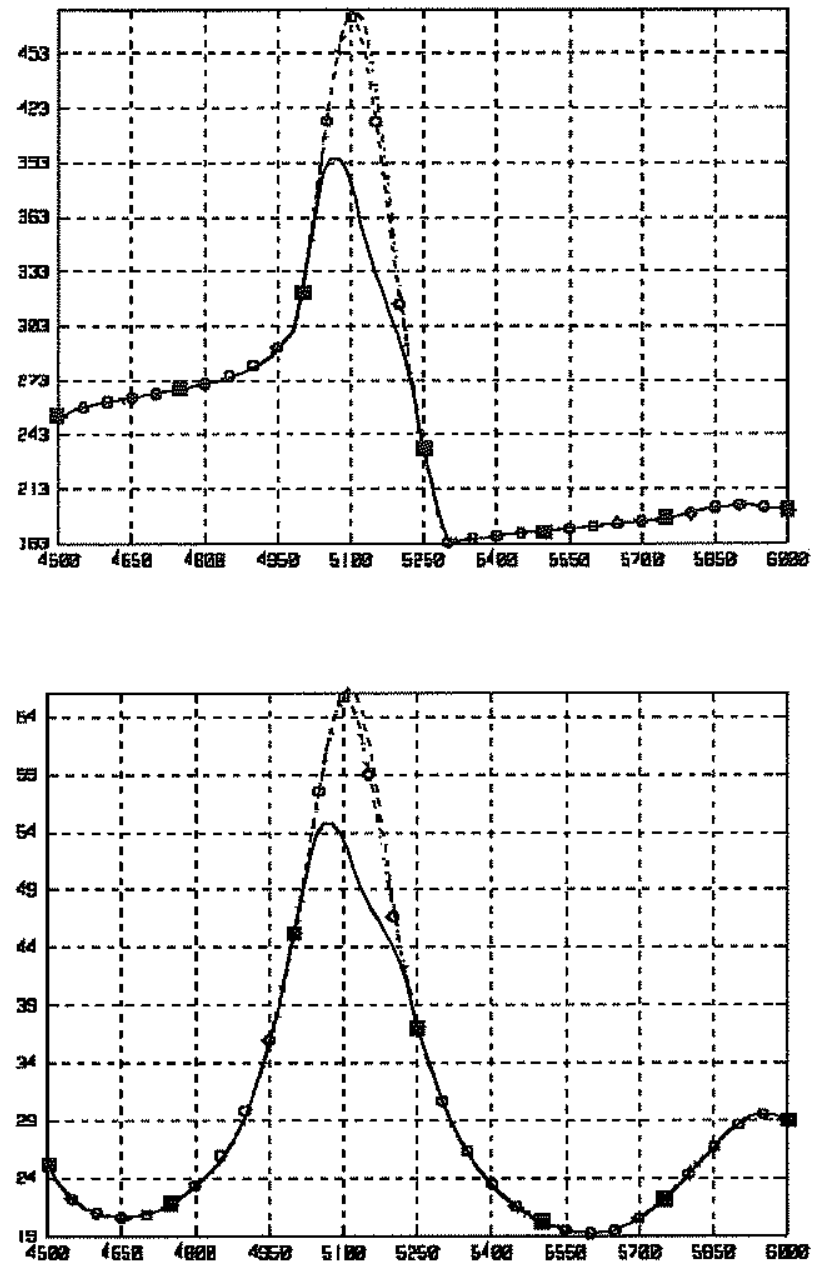

FIG. 3

Top : average of the highest values of the current flow modulus square (here 20)

Bottom : quadratic average of all the values of the modulus square of the current flows versus frequency $(4.5-6.0 \mathrm{GHz})($ see text)

\subsection{The dielectric cube}

We consider a dielectric cube (100 mm by side) simulated with 7920 surface finite elements. The electric dipole is located at the center of the cube and oriented along one of its axis.

Using our method, we obtain some resonance frequencies (TM modes) as we can observe it in the figure 4 (level 2). We can determine a first set of resonance frequencies. In order to verify our theoretical results, we calculate the values of these resonance frequencies with the well-known analytic formula [14]. We also simulate this structure with a FD-TD code (figure 5). We observe these resonance frequencies are quitely the same. The dermination of the resonance frequencies of a dielectric cube is difficult. However, our method can determine quickly enough without difficulties.

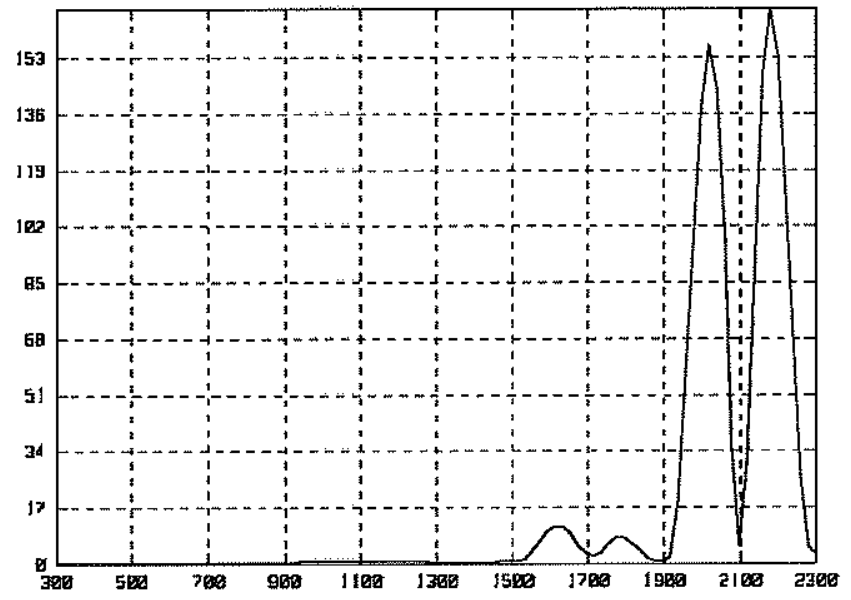

Level 0 : starting with an uniform set of 10 points on the frequency band.

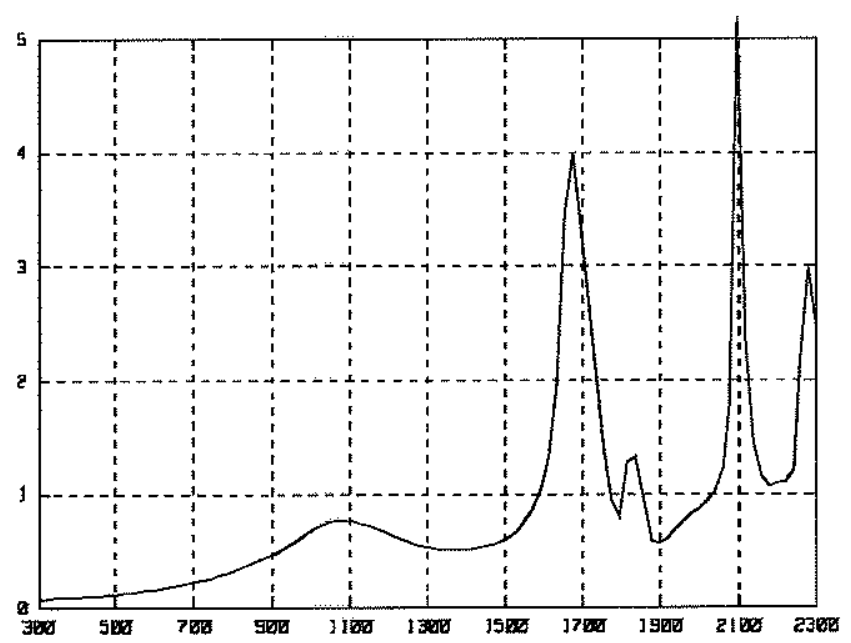

Level 1 : automatic refinement with 9 non equidistant additional points.

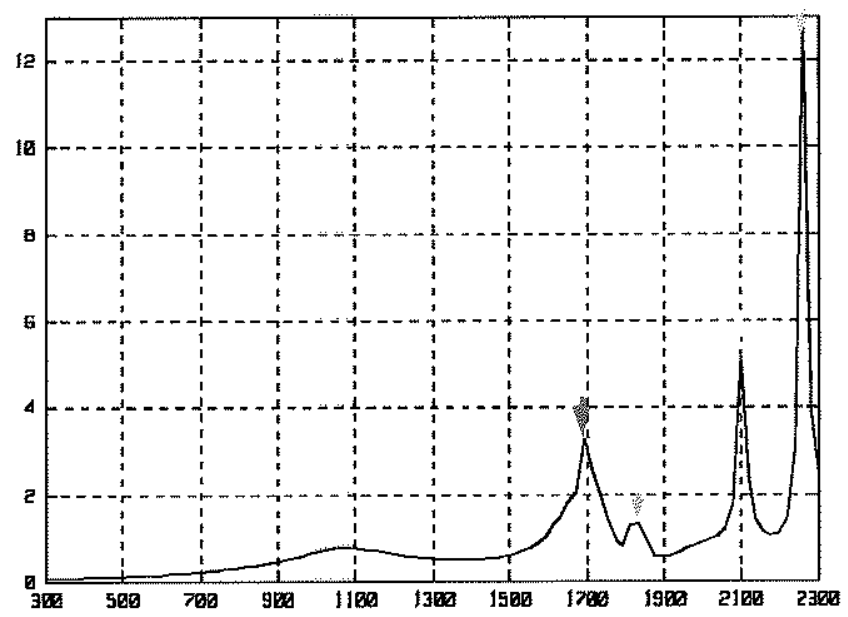

Level $2:$ a new refinement with 8 other adding points

FIG. 4: Automatic determination of the resonance frequencies of dielectric structure $\left(\varepsilon_{\mathrm{r}}=10\right)$.

$(0.3-2.3 \mathrm{GHz})$ 


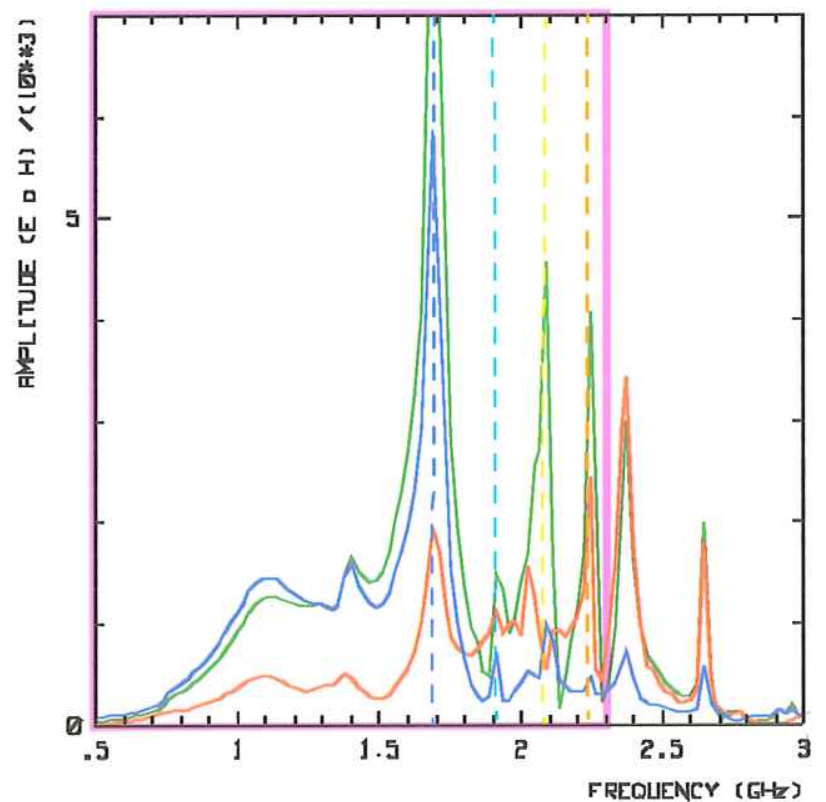

FIG. 5 : Variations of the six components of the electromagnetic field obtained with a FD-TD technique.

\section{Conclusion}

In this paper, we have proposed an original and fast autoadaptive optimization technique to calculate the electromagnetic behavior of an 3D antenna over a large frequency band with a very few number of frequency samples. It is based on the knowledge of the formal derivatives of the current flow and the use of the integral reaction coupling with an accurate polynomial interpolation algorithm. So, our SR3D solver is now able to compute complex $3 \mathrm{D}$ radiating structures with accuracy and time saving. Comparisons between our results and those obtained by other methods are done and we observe an excellent agreement.

\section{References}

[1] J. Yeo, R. Mittra, An algorithm for interpolating frequency variations of MoM matrices arising in the analysis of planar microstrip structures, IEEE Trans. Microw. Theory Tech., vol.51, n³, 1018-1025, March 2003.

[2] J.C. Rautio, Planar Electromagnetic Analysis, IEEE Microw. Magazine,vol.3, 35-41, 2003.

[3] E. Meijering, A chronology of interpolation : from ancient astronomy to modern signal and image processing, Proc. IEEE, 90, vol.3, 319-342, 2002.

[4] L. Xia, C.-F. Wang, L.-W. Li, P.-S. Kooi, M.-S. Leong, Fast characterization of microstrip antenna resonance in multilayered media using interpolation/extrapolation methods, Microw. Opt.
Technol. Lett., vol.28, n5, 342-346, 2001.

[5] R. Lehmensiek, P. Meyer, Creating accurate multivariate rational interpolation models of microwave circuits by using efficient adaptive sampling to minimize the number of computational electromagnetic analyses, IEEE Trans. Microw. Theory Tech., vol.49, n8, 14191430, 2001.

[6] J.-L. Hu, C. H. Chan, T. K. Sarkar, Optimal simultaneous interpolation/extrapolation algorithm of electromagnetic responses in time and frequency domains, IEEE Trans. Microw. Theory Tech., vol.49, $\mathrm{n}^{\circ} 10,1725-1732,2001$.

[7] B. Jiang, T. Su, H. Ling, Frequency interpolation of electromagnetic scattering data using a hybrid model, Microw. Opt. Tech. Lett., vol.27, n5, 307-312, December 2000.

[8] S. Narayana, T. K. Sarkar, R. Adve, M. Wicks, V. Vannicola A Comparison of Two Techniques for the Interpolation/Extrapolation of Frequency Domain Responses, Digital Signal Processing, 6, 1, 51-67, 1996.

[9] P.L.Butzer and R.L.Stens, Sampling theory for notnecessarily band-limited functions: A historical overview, SIAM Review, vol.34, pp.40-53, 1992.

[10] X. Yang, E. Arvas, Use of frequency-derivative information in two-dimensional electromagnetic scattering problems, IEE Proc -Microw. Antennas Propag.(Part. H), vol.138, n²4, 269-272, 1991.

[11] P. Dubois, C. Dedeban, J.P. Zolesio, J.P. Damiano, Use of the frequency derivative of the harmonic regime for sensibility analysis, European Congress on Computational Methods in Applied Sciences and Engineering - ECCOMAS 2004 - IFIP 7.2 "Moving Boundary and Control", Jyväskylä, Finland, 24-28 July 2004, to be published in 2005.

[12] J.-P. Marmorat, J.-P. Zolésio, Contract Report Armines-France Télécom, September 2000.

[13] P. Ratajczak, P. Brachat, J.-L. Guiraud, "Rigorous analysis of three-dimensional structures incorporating dielectrics", IEEE Trans. Antennas Propag., vol.42, n8, 1077-1088, 1994.

[14] C. Balanis, Antenna theory, $2^{\text {nd }}$ ed., J. Wiley, 1997. 